\title{
Announcing the winner of the 2010 Kurt Jellinger Prize
}

\author{
Werner Paulus
}

Published online: 18 July 2010

(C) Springer-Verlag 2010

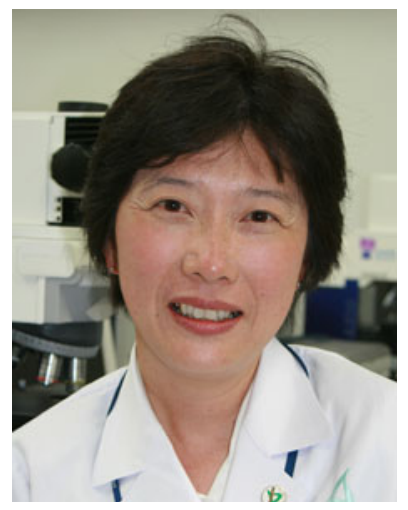

Dr. Yukiko Shishido-Hara is the winner of the 2010 Kurt Jellinger Prize for Excellence in Scientific Writing, based on the review article "Progressive multifocal leukoencephalopathy and promyelocytic leukemia nuclear bodies: a review of clinical, neuropathological, and virological aspects of JC virus-induced demyelinating disease", published in this issue of Acta Neuropathologica and reflecting the author's experience in both neuropathology and virology.

Yukiko Shishido-Hara has been engaged in the study of JC virus since early research as a fellow at the National Institutes of Health, Tokyo and the Tokyo Metropolitan Institute for Neuroscience (1992-1995), where she examined the expression of $\mathrm{JC}$ virus proteins in insect cells using Baculoviral vectors and in COS7 cells using the SR $\alpha$ promoter. From 1995 to 1999, she continued her studies at the NIH, USA, by examining JC virus in AIDS patients,

W. Paulus $(\bowtie)$

Institute of Neuropathology, University Hospital Münster, Domagkstr. 19, 48149 Münster, Germany

e-mail: werner.paulus@uni-muenster.de and by determining the complete sequence of JC virus Tokyo-1 strain. After moving back to Tokyo, she has explored a variety of projects related to JC virus, including the induction of oligodendroglial cells from cultured neural stem cells, the nuclear structure of JC virus-infected human oligodendroglial cells, the role of JC virus in brain tumors, and the pathogenic role of promyelocytic leukemia nuclear bodies (PML-NBs) as an intranuclear target of JC virus infection, which represents a major part of her Prize winning paper. Yukiko Shishido-Hara earned MD and $\mathrm{PhD}$ degrees, and she underwent training in both virology and neuropathology before obtaining certification in pathology. Currently, she serves as surgical pathologist and Assistant Professor, Department of Pathology, Kyorin University School of Medicine, Tokyo and as Visiting Scientist, Tokyo Metropolitan Institute for Neuroscience.

The 2010 Kurt Jellinger Prize includes the invitation to the 17th International Congress of Neuropathology in September 2010 (ICN 2010) organized by Herbert Budka and Hans Lassmann from Vienna, a capital city of neuropathology where Acta Neuropathologica has been founded almost 50 years ago and edited for 43 years by Franz Seitelberger und Kurt Jellinger. The Prize also includes the invitation to join the Editorial Board which represents a group of enthusiastic scientists wishing to provide the best service to the neurocommunity. The present issue includes review papers written by speakers of ICN 2010. To make these and other papers as topical as possible, processing of papers is very fast in this journal. For example, the mean time from submission to first decision has been 12 days in 2010, and it was 3 days for the ICN 2010 papers included in this issue. This has been possible only thanks to a dynamic Editorial Board which is glad to have Yukiko Shishido-Hara and her expertise aboard. 